\title{
Trans-Thoracic Echocardiographic Aortic Blood Flow Peak Velocity Variation, Distance Minute, Aortic Velocity Time Integral and Postoperative Outcome in Pediatric Surgical Patients-An Observational Pilot Study Protocol
}

\author{
Claudine Kumba ${ }^{1,2}$ (1) \\ ${ }^{1}$ Department of Pediatric Anesthesia and Critical Care, Necker Enfants Malades University Hospital, Assistance Publique \\ Hôpitaux de Paris, APHP, University of Paris, Paris, France \\ ${ }^{2}$ Ecole Doctorale 563 Médicament-Toxicologie-Chimie-Imageries (MTCI), Université de Paris, Paris, France \\ Email: claudine.kumba@gmail.com
}

How to cite this paper: Kumba, C. (2020) Trans-Thoracic Echocardiographic Aortic Blood Flow Peak Velocity Variation, Distance Minute, Aortic Velocity Time Integral and Postoperative Outcome in Pediatric Surgical Patients-An Observational Pilot Study Protocol. Open Journal of Internal Medicine, 10, 90-95.

https://doi.org/10.4236/ojim.2020.101009

Received: February 2, 2020

Accepted: March 15, 2020

Published: March 18, 2020

Copyright $\odot 2020$ by author(s) and Scientific Research Publishing Inc. This work is licensed under the Creative Commons Attribution International License (CC BY 4.0).

http://creativecommons.org/licenses/by/4.0/

\begin{abstract}
Background: A Randomized Controlled Trial (RCT) has been elaborated where goal directed fluid and hemodynamic therapy (GDFHT) will be realized with trans-thoracic echocardiographic aortic blood flow peak velocity variation $(\Delta$ Vpeak) and distance minute $(\mathrm{DM})$ to guide fluid therapy and hemodynamics in high risk pediatric surgical patients. This RCT will clarify the impact of GDFHT with $\triangle$ Vpeak and DM on postoperative outcome in terms of morbidity, length of stay in the intensive care unit (LOSICU), length of mechanical ventilation (LMV) and length of hospital stay (LOS) in children. To determine values of $\Delta$ Vpeak, DM and VTI predictive of these postoperative outcomes, an observational pilot study will be realized. This pilot study is described here. The primary objective of this study is to determine values of $\Delta V$ peak, DM and ITV predictive of postoperative outcome in children in terms of morbidity. The secondary objectives are to determine values of $\Delta$ Vpeak, DM and ITV predictive of LOSICU, LMV, LOS, intraoperative, postoperative fluid administration and vasoactive-inotropic therapy. Methods: 500 - 1000 children aged less than 18 years will be included prospectively. Statistic analysis will be realized with XLSTAT 2019.4.2 software or plus. Results and Conclusions: This trial protocol will determine values of $\Delta V$ peak, DM and ITV with echocardiography predictive of postoperative outcome in children.
\end{abstract}

\section{Keywords}

Children, Aortic Blood Flow Peak Velocity Variation, Aortic Velocity Time 
Integral, Distance Minute, Postoperative Outcome, Pilot Study Protocol, Trans-Thoracic Echocardiography

\section{Introduction}

A recent retrospective observational study in pediatric surgical patients in neurosurgery, abdominal surgery and orthopedic surgery revealed that postoperative outcome in terms of morbidity was multifactorial [1]. This study also revealed that the rate of patients with postoperative complications in terms of organ dysfunction in this cohort of more than 594 children was $23 \%$. To implement improvement measures on this incidence, GDFHT protocols with transthoracic echocardiography have been elaborated [2]-[6]. Intraoperative goal directed fluid and hemodynamic therapy in adults has demonstrated reduction in postoperative complications [7]. In pediatric patients, it has not yet been established that GDFHT improved postoperative outcome. The issue in pediatrics is that validated hemodynamic parameters for GDFHT remain limited. Indices like $\Delta$ Vpeak, DM velocity time integral (VTI) have been validated in several studies in children [8]-[12]. $\Delta$ Vpeak has been validated for fluid responsiveness in pediatric surgical patients [8] [9] [10] [11]. Stroke volume (SV) can be calculated with the following formula SV $=\mathrm{VTI} \times \mathrm{D}^{2} \times \mathrm{II} / 4$ [10]. Cardiac output $(\mathrm{CO})$ can be calculated with this formula $\mathrm{CO}=\mathrm{VTI} \times \mathrm{D}^{2} \times \mathrm{II} / 4 \times \mathrm{HR}$ [13]. Where $\mathrm{D}$ is the diameter of the aortic annulus in the parasternal longitudinal view and HR is the heart rate. VTI can reflect $\mathrm{CO}$, however, in children, it is recommended to use distance minute (DM) since cardiac output is heart rate dependent [12]. DM can be calculated as follows DM $=$ VTI $\times$ HR [12]. What has not been realized is to determine the impact of $\triangle \mathrm{Vpeak}$, DM, VTI in GDFHT on postoperative morbidity, LOSICU, LMV, LOS, fluid administration and vasoactive inotropic therapy. A RCT has been elaborated where transthoracic echocardiography with $\Delta$ Vpeak, DM velocity time integral (VTI) will be integrated in GDFHT to clarify their impact on postoperative outcome in children since this has not yet been realized [6]. What has not been realized either is to determine values of $\triangle$ Vpeak, DM and VTI predictive of outcome in terms of postoperative morbidity, LOSICU, LMV, LOS and intraoperative and postoperative fluid administration and vasoactive inotropic therapy. An observational pilot study is described here which has the primary objective to determine values of $\Delta V$ peak, DM and ITV predictive of postoperative outcome in children in terms of morbidity. The secondary objectives of this pilot observational trial described here are to determine values of $\Delta$ Vpeak, DM and ITV predictive of postoperative length of stay in the intensive care unit, length of mechanical ventilation, length of hospital stay; intraoperative, postoperative fluid administration and vasoactive-inotropic therapy.

The primary outcome measures will be postoperative organ dysfunction until discharge from hospital. 
The secondary outcome measures will be the number of postoperative days spent in the intensive care unit (ICU), the number of postoperative days spent on invasive or non-invasive mechanical ventilation, the number of postoperative days spent in the conventional hospitalization ward, the quantity of intraoperative fluid therapy and vasopressor-inotropic score (VIS).

This study is part of the Thesis entitled "Do goal directed therapies improve postoperative outcome in children? (Perioperative Goal Directed Fluid and Hemodynamic Therapy; Transfusion goal directed therapy using viscoelastic methods and enhanced recovery after surgery and Postoperative outcome)" [14]-[18]. This Thesis is registered at http://www.theses.fr/s232762.

\section{Methods and Materials}

This study has been registered at the French National Agency on Medication and Drug Safety (ANSM, Agence Nationale de Sécurité du Médicament et des Produits de Santé) under the number ID RCB: 2020-A00266-33. After approval by the Ethics Committee (which will be determined and precised later) and parents' information, patients will be included prospectively in one cohort. Patients will be managed as usual or at the discretion of the medical doctor in charge in terms of anesthesia. $\triangle$ Vpeak, DM and VTI will be measured with trans-thoracic echocardiography twice intraoperatively after induction of anesthesia and at the end of surgery. These parameters will be assessed in the apical five-chamber view. $\Delta$ Vpeak will be calculated as follows [(Vpeak maximum - Vpeak minimum $) /\{($ Vpeak maximum + Vpeak minimum $) / 2\}] \times 100$ [11]. DM will be calculated as follows DM $=$ VTI $\times$ HR where VTI is aortic velocity time integral and HR heart rate [12].

Inclusion criteria are patients under 18 years old scheduled for any type of surgery where fluid therapy with crystalloids or colloids will be administered intraoperatively. Exclusion criteria are patients more than 18 years old, parents or patient's refusal.

Since echocardiography is operator dependent, it will be realized by the same person experienced in echocardiography to reduce the inter-observer variability. Parameters such as age, gender, type of surgery, elective or urgent surgery, American Society of Anesthesiologists status (ASA), weight, height, prematurity, blood pressure, heart rate, pulse oxymetry, hemoglobin levels, platelet count, leucocyte count, activated thromboplastin, prothrombin time, fibrinogen, blood urea nitrogen, serum creatinin levels, C-reative protein levels (CRP), procalcitonin (PCT) levels, hepatic functional tests will be registered (if analysed).

Preoperatively basal values of blood pressure, heart rate, core temperature, pulse oxymetry, will be registered prior to anesthesia and surgery and intraoperatively hourly. Intraoperative parameters registered will be blood product transfusion (packed red blood cells (PRBC), fresh frozen plasma (FFP), concentrated platelet units (CUP), fibrinogen, cryoprecipitate, concentrated complex of prothrombin (CPP) or other blood product derivatives, crystalloids and colloids or 
other fluids administered, blood loss, urinary output, quantity of inotrops administered and mechanical ventilation parameters, regional cerebral oxygen saturation, renal oxygen saturation and mixed venous oxygen saturation (if monitored) and lactate levels (if monitored).

Postoperative parameters registered will be blood pressure, heart rate, core temperature, pulse oxymetry, mixed venous oxygen saturation ( $\mathrm{ScVO} 2)$, lactate levels, cerebral $(\mathrm{ScO} 2)$ and renal oxygen saturation $(\mathrm{SrO} 2)$, blood product transfusion (PRBC, FFP, CUP), fibrinogen, cryoprecipitate, concentrated complex of prothrombin other blood product derivatives, crystalloids, colloids or other fluids administered, blood loss, urinary output, quantity of inotrops admnistered, mechanical ventilation parameters, hemoglobin, platelet, leucocyte levels, CRP, PCT, hepatic functional tests, blood urea nitrogen, serum creatinin levels (if monitored and analysed).

Statistic analysis will be realized with XLSTAT 2019.4.2 or plus software. Normally distributed and non-normally distributed variables will be compared using Student t-test or Mann-Whitney test and Wilcoxon test or Kruksal-Wallis test respectively. Normally distributed variables will be expressed in terms of means with standard deviation. Non-normally distributed variables will be expressed in terms of medians with interquartile ranges. Categorical variables will be compared with the exact Fisher's test or Chi-squared test accordingly. Categorical variables will be expressed as percentages with $95 \%$ confidence intervals. To assess for independent predictors of adverse postoperative outcome, multivariate analysis will be realized. A P-value $\leq 0.05$ will be considered significant. Missing data will not be included.

The number of patients included will be between 500 and 1000 patients. This number was determined after the results of the retrospective study [1] which evidenced a postoperative complication rate of $23 \%$ in 594 children. The issue was to have a similar population in size. Patients will be categorized according to age in premature neonates (gestational age less than 37 weeks), term neonates (less than one-month-old), children aged from one month to one year, children aged from one to three years, children aged from three to six years, from six to ten years and from ten to less than 18 years.

The study will be multicentric.

\section{Discussion, Results and Conclusions}

GDFHT has proven its efficiency in terms of decreased mortality and morbidity in adults. In children, this has not yet been established. Parameters of GDFHT applied in adults like pulse pressure variation remain controversial in children. $\Delta$ Vpeak has been validated in children for fluid responsiveness. $\Delta$ Vpeak, DM and VTI values predictive of postoperative outcome in terms of morbidity, LOSICU, LMV and LOS have not yet been established. This observational study protocol will clarify values of $\Delta \mathrm{Vpeak}, \mathrm{DM}$ and VTI predictive of postoperative morbidity, LOSICU, LMV, LOS, intraoperative and postoperative fluid therapy 
and vasopressor-inotropic therapy in children admitted for surgery. A randomized controlled trial will determine the impact of GDFHT with $\triangle$ Vpeak, DM and VTI on postoperative outcome in pediatric patients admitted for major surgery since this has not yet been demonstrated in children.

\section{Acknowledgements}

The abstract of this manuscript has been submitted to the European Congress for Paediatric Anaesthesiology ESPA 2020.

\section{Conflicts of Interest}

The author declares no conflict of interest.

\section{References}

[1] Kumba, C., Cresci, F., Picard, C., Thiry, C., Albinni. S. and Orliguet, G. (2017) Transfusion and Morbi-Mortality Factors: An Observational Descriptive Retrospective Pediatric Cohort Study. Journal of Anesthesia \& Critical Care: Open Access, 8 , Article ID: 00315. https://doi.org/10.15406/jaccoa.2017.08.00315

[2] Kumba, C., Raisky, O., Bonnet, D. and Tréluyer, J.M. (2019) Perioperative Echocardiographic Hemodynamic Parameters and Postoperative Outcome in Pediatric Congenital Heart Disease: A Descriptive Observational Prospective Pilot Study Protocol. International Journal of Pediatrics \& Neonatal Care, 5, 160.

[3] Kumba, C., Raisky, O., Bonnet, D. and Treluyer, J.M. (2019) Perioperative Goal Directed Fluid and Hemodynamic Therapy with Echocardiography in Pediatric Congenital Heart Disease: A Study Protocol. EC Paediatrics, 8, 1-7.

[4] Kumba, C. and Tréluyer, J.M. (2019) Perioperative Echocardiographic Hemodynamic Parameters and Postoperative Outcome in Pediatric Surgical Patients: A Descriptive Observational Prospective Pilot Study Protocol. Research in Pediatrics \& Neonatology, 4, RPN.000580.

[5] Kumba, C., et al. (2020) Perioperative Goal Directed Fluid and Hemodynamic Therapy with Echocardiography in Children: A Study Protocol. Research in Pediatrics \& Neonatology, 4, RPN.000583.

[6] Kumba, C., et al. (2020) Goal Directed Fluid and Hemodynamic Therapy and Postoperative Outcomes in Children Value of Trans-Thoracic Echocardiographic Aortic Bloof Flow Peak Velocity Variation: A Multicentre Randomized Controlled Trial Protocol. Advances in Pediatric Research, 7, 35. https://doi.org/10.35248/2385-4529.20.7.35

[7] Chong, M., Wang, Y., Berbenetz, N., et al. (2018) Does Doal-Directed Haemodynamic and Fluid Therapy Improve Peri-Operative Outcomes? A Systematic Review and Meta-Analysis. European Journal of Anaesthesiology, 35, 469-483. https://doi.org/10.1097/EJA.0000000000000778

[8] Favia, I., Romagnoli, S., Di Chiara, L. and Zaccaria, R. (2017) Predicting Fluid Responsiveness in Children Undergoing Cardiac Surgery after Cardiopulmonary Bypass. Pediatric Cardiology, 38, 787-793. https://doi.org/10.1007/s00246-017-1582-0

[9] Desgranges, F.P., Desebbe, O., Pereira de Souza Neto, E., Raphael, D. and Chassard, D. (2016) Respiratory Variation in Aortic Blood Flow Peak Velocity to Predict Fluid Responsivenes in Mechanically Ventilated Children: A Sytematic Review and Meta-Analysis. Pediatric Anesthesia, 26, 37-47. https://doi.org/10.1111/pan.12803 
[10] Morparia, K.G., Reddy, S.K., Olivieri, L.J., Spaeder, M.C. and Schuette, J.J. (2018) Respiratory Variation in Peak Aortic Accurately Predicts Fluid Responsiveness in Children Undergoing Neurosurgery Under General Anesthesia. Journal of Clinical Monitoring and Computing, 32, 221-226. https://doi.org/10.1007/s10877-017-0013-3

[11] Pereira de Souza Neto, E., Grousson, S., Duflo, F., Ducreux, C., Joly, H., Convert, J., et al. (2011) Predicting Fluid Responsiveness in Mechanically Ventilated Children Under General Anaesthesia Using Dynamic Parameters and Transthoracic Echocardiography. British Journal of Anaesthesia, 106, 856-864.

https://doi.org/10.1093/bja/aer090

[12] Solinski, A., Klusmeier, E., Horst, J.P., Korperich, H., Haas, N.A., Kececioglu, D., et al. (2018) Centile Curves for Velocity-Time Integral Times Heart Rates as a Function of Ventricular Length: The Use of Minute Distance Is Advantageous to Enhance Clinical Reliability in Children. Journal of the American Society of Echocardiography, 31,105-112. https://doi.org/10.1016/j.echo.2017.09.011

[13] Klimczak, C. (2010) Echocardiographie Clinique. 6th Edition, Elsevier Masson, Paris, 32-34.

[14] Kumba, C. and Mélot, C. (2019) The Era of Goal Directed Therapies in Paediatric Anaesthesia and Critical Care. EC Emergency Medicine and Critical Care, 3, 306-309.

[15] Kumba, C. (2019) Do Goal Directed Therapies Improve Postoperative Outcome in Children? (Perioperative Goal Directed Fluid and Hemodynamic Therapy; Transfusion Goal Directed Therapy Using Viscoelastic Methods and Enhanced Recovery After Surgery and Postoperative Outcome): A Study Research Protocol. Acta Scientific Paediatrics, 2, 17-19. https://doi.org/10.31080/ASPE.2019.02.0094

[16] Kumba, C. (2019) Future Evolution of Intraoperative Goal Directed Fluid and Hemodynamic Therapy in Children. Advances in Pediatric Research, 6, 29. https://doi.org/10.35248/2385-4529.19.6.29

[17] Kumba, C., et al. (2019) A Systematic Review and Meta-Analysis of Intraoperative Goal Directed Fluid and Haemodynamic Therapy in Children and Postoperative Outcome. Journal of Emergency Medicine \& Critical Care, 5, 9. https://doi.org/10.13188/2469-4045.1000020

[18] Kumba, C. (2020) Innovating Applications with Trans-Thoracic Echocardiography in Goal Directed Fluid and Hemodynamic Therapy in Children. EC Clinical and Medical Case Reports, 3, 1-3. 